\title{
FIR Infrared Emission as Indicator of Interaction in Hickson Compact Groups
}

\author{
P. Merluzzi, A. A. Shaker ${ }^{1}$ and G. Longo \\ Osservatorio Astronomico di Capodimonte, Napoli, Italy
}

\section{Introduction}

Due to their very high spatial density and relatively low velocity dispersion, Hickson's compact groups of galaxies (hereafter HCGs, Hickson et al. 1989a, $\mathrm{H} 89 \mathrm{a}$ ) are ideal test grounds where to study the physics of galaxy interaction.

Tidal shocks induced by galaxy interaction are expected to trigger rapid bursts of star formation in the circumnuclear region of galaxies. The increases in density and temperature caused locally by such shocks produce an enhancement in both the total FIR luminosity and in the ratio between the FIR fluxes at 60 and $100 \mu \mathrm{m}$. These enhancements have been actually observed in the circumnuclear regions of both interacting and "putative" merging galaxies and should therefore be found also in all galactic environments where the frequency of interaction is higher than in the field. However, while interactions are more frequent among HCGs members than in the field (e.g. Hickson 1994, Shaker et al. 1999), there are conflicting arguments on whether HCGs present or do not present a FIR excess. As stressed by many authors, the critical point is the IRAS data resolution which prevents to map the spatial distribution of the FIR emission. We investigate the FIR properties of a selected sample of HCGs by using the new high spatial resolution data from Allam et al. 1996 (A96). In particular, we address the problem whether or not there is any FIR enhancement in the compact groups and the reasons of that. This is a short presentation of the analysis performed and of the results obtained (Merluzzi et al. 1999).

\section{The sample}

The sample selection criteria and the details of the present analysis are described in Merluzzi et al. (1999). We divided A96 data into two samples. In the first sample, which contains 39 resolved galaxies (RGs), the FIR emission comes from isolated galaxies only, while the second sample contains 19 unresolved groups (UGs) where the FIR flux comes from more than one galaxy not resolved by IRAS. All the objects were detected both at 60 and $100 \mu \mathrm{m}$.

We collected and derived from the literature different properties of the groups and of the resolved galaxies (the mean redshift of the groups and the morphological types of the galaxies, the distances, the optical luminosity, the molecular gas content, the radio emission). Furthermore, from the FIR fluxes

${ }^{1}$ NRIAG, Helwan, Cairo, Egypt 
by A96, we computed the FIR color temperatures, the FIR luminosity, the dust mass and the star formation rate (SFR).

\section{Results}

The FIR fluxes. We find that while $35 \%$ of the Hickson et al. $(1989, \mathrm{H} 89 \mathrm{~b})$ galaxies have a FIR flux ratio greater than 0.4 , for the A96 galaxies this percentage is reduced to $20 \%$. This is particularly relevant since starburst galaxies usually have flux ratios greater than 0.4 (Young et al. 1989). This discrepancy is very likely due to the low accuracy of IRAS-PSC data used by H89b in providing fluxes for extended objects. (Many objects which were resolved by IRAS were erroneously included in the PSC). Therefore, H89b overestimated the number of galaxies in HCGs which were interpreted as strongly interacting.

Resolved and unresolved HCGs. Since HCGs are mainly nearby systems, the lack of resolution may be due either to their distance or to compactness. We found that the averages of the FIR properties for the two samples are consistent. By studying the distributions of the FIR quantities for the two samples we found no differences between the temperature distribution of the RGs and the UGs. On the other hand, the distribution of the UGs is clearly shifted towards higher values of the FIR luminosity with respect to the similar distribution of the RGs. As to the dust mass, we find a wide range of values for the UGs while RGs are clearly peaked at lower values. These results are confirmed by applying the Kolmogorov-Smirnov (K-S) test. The natural conclusion is that even though some groups cannot be resolved due to their high distance and/or compactness, they are still extracted from the same population. Some of UGs have higher luminosity and dust mass since they result from the sum of many individual contributions.

Comparison with other galaxy samples. As templates we used three different samples of 35 isolated, 46 interacting (Bushouse et al. 1988) and 43 Markarian galaxies (Mazzarella et al. 1991) detected at both 60 and $100 \mu \mathrm{m}$. The comparison between high resolution FIR data of the HCGs and the low resolution FIR data of the comparison samples is allowed since FIR fluxes are in all cases integrated on the whole galaxies. The average FIR properties of RGs are consistent, within the errors, with each of the three samples. Dust temperature, flux ratio and SFR for the RGs seem to be closer to the corresponding quantities for the isolated galaxies, but, due to the large standard deviation, it is not possible to demonstrate any peculiar similarity between the RGs and the isolated galaxies rather than between the RGs and the interacting or the Markarian galaxies. The FIR luminosity distribution of RGs appears to be intermediate between those of the isolated and interacting galaxies in agreement with Zepf (1993). It seems that some galaxies in HCGs are experiencing interaction, while other systems are quiescent and present a FIR luminosity similar to that of the isolated galaxies. This fact is confirmed by the K-S analysis.

\section{Discussion}

From the previous analysis, we conclude that HCGs do not show evidence for any FIR excess and, therefore, they should form a sample of not interacting 
or mildly interacting galaxies. On the other hand, by studying the relations between FIR emission and the emission at other wavelengths, we found for the RGs that FIR emission correlates with the optical luminosity, the $\mathrm{CO}$ and the radio emission.

FIR and optical luminosities correlate for the RGs following an exponential law which is in agreement with the relations obtained by other authors for galaxies in HCGs (Verdes-Montenegro et al. 1998) for isolated galaxies (Perea et al. 1997, Karachenseva et al. 1973) and for a sample of elliptical galaxies (Merluzzi 1998). If the FIR radiation from a galaxy can be used to estimate the current star formation and the blue light estimates instead the past star formation rate in the galaxy, the previous result indicates that HCGs, isolated and elliptical galaxies present similar relations between current and past star formation.

We used the CO data by Leon et al. (1998), who observed an enhancement of the molecular gas content in HCGs galaxies, and found a correlation between the molecular gas mass and both the dust mass and the FIR luminosity. Using the 27 galaxies in common between their and our sample, we confirm that, within the errors, the molecular gas is linearly correlated with both the FIR luminosity and the dust mass. The physical explanation of this fact is that the two components of the ISM are both related to the star formation. Leon et al. (1998) also found that the molecular gas is located in the center of the galaxies and suggested that "tidal interactions can drive the gas component inwards, by removing its angular momentum, and concentrating it in the dense central regions ...". This explanation was also used by Menon (1995) who found that the radio emission is mainly concentrated in the central region of the galaxies in HCGs. These two correlations strongly suggest that also the FIR emission comes from the central regions.

Taking into account the comparisons between FIR emission and emissions at other wavelengths in HCGs, it is, therefore, reasonable to consider the FIR emission as originating mainly from star formation induced by interaction. This conclusion is not due to the observations of a clear FIR enhancement but to the presence of the correlations between the FIR emission and peculiar conditions of the ISM in the center of the galaxies which are usually related to the presence of interactions. Therefore, the lack of FIR enhancement does not question the physical reality of the groups as suggested by Sulentic \& de Mello Rabaça (1993) but rather puts under discussion the goodness of the FIR emission as a SFR indicator.

Hashimoto et al. (1998, H98) investigated how star formation rates within galaxies vary with environment by using a large galaxy sample (see H98 for details). They found a general depletion of SFR with the local density enhancement in different galaxy populations (field, clusters, rich groups, etc.). Moreover, when they further distinguished among "rich" and "poor" clusters (see below), they found in the rich ones a lower level of normal star formation (due to the galaxy internal evolution) and starbursts, and in the poor clusters an enhanced level of both. Then, the question is the following: are HCGs more similar to rich or poor clusters?

Following the criteria of $\mathrm{H} 98$ we used the the total luminosity, $\mathrm{L}_{T}$, in $\mathrm{R}$ band to investigate the "richness" of HCGs. We used the data by H89a to compute the total $\mathrm{R}$ luminosity of 46 HCGs. Taking into account the ranges 
of $\mathrm{L}_{T}$ defined by $\mathrm{H} 98$ to disentangle between rich and poor clusters, we found that HCGs are a non-homogeneous data set of rich and poor clusters which are experiencing different environmental effects and, therefore they present different levels of star formation and FIR emission. This can explain the lack of an overall enhancement in FIR emission. On the other hand, since the histogram of $\mathrm{L}_{T}$ is Gaussian (D.L. Tucker, private communication), we further used the peak value of the distribution to separate poor from rich clusters. With this convention, $67 \%$ of the groups are rich, leading us to conclude that HCGs are mainly rich clusters which, in agreement with $\mathrm{H} 98$, present a reduced level of star formation. In fact, in these systems, which are dense and permeated by a hot intergalactic medium, the ram pressure and the evaporative stripping of gas are very efficient. Therefore, during a past interaction the galaxies of a group must have had a rapid increase of the star formation which reduced the amount of the gas inducing a subsequent depletion of the long term star formation.

Acknowledgments. We thank D.L. Tucker and S.S. Allam for the useful discussions. We are grateful to the meeting organizers.

\section{References}

Allam, S., Assendrop, R., Longo, G., Braun, M., \& Richter, G. 1996, A\&A, S., 117, 39 (A96)

Bushouse, H. A., Lamb, S. A., \& Werner, M. W. 1988, ApJ, 335, 744

Hashimoto, Y., Oemler, A. Jr., Lin, H., \& Tucker, D. L. 1998, ApJ, 499, 589, (H98)

Hickson, P. 1994, Atlas of Compact Groups of Galaxies, Gordon and Breach Science Publishers

Hickson P., Kindl E., \& Auman J. R. 1989a, ApJS, 70, 687 (H89a)

Hickson, P., Menon, T. K., Palumbo G. C. G., \& Persic, M. 1989, ApJ, 341, 679 ( $\mathrm{H} 89 \mathrm{~b})$

Karachenseva, V. E., Lebedev, V. S., \& Shcherbanovskij, A. L. 1973, Comm. of Special Astrophys. Obs. USSR 8

Leon, S., Combes, F., \& Menon T. K. 1998, A\&A, 330, 37

Mazzarella, J. M., Bothun, G.D., \& Boroson, T. A. 1991, AJ, 101, 2034

Menon, T. K. 1995, AJ, 110, 6

Merluzzi, P. 1998, A\&AS., 338, 807

Merluzzi, P., Shaker, A. A., \& Longo, G. 1999, submitted

Perea, J., del Olmo, A., Verdes-Montenegro, L., \& Yun, M. S. 1997, ApJ, 490, 166

Shaker, A. A., Longo, G., \& Richter, G. 1999, Mem. SAIt, in press

Sulentic, J. W., \& de Mello Rabaça D. 1993, ApJ, 410, 520

Verdes-Montenegro, L., Yun, M. S., Perea, J., del Olmo, A., \& Ho, P. T. P. 1998, ApJ, 497, 89

Young, J. S., Xie, S., Kenney, J. D. \& Rice, W. L. 1989, ApJS, 70, 699

Zepf, S. E., 1993, ApJ, 407, 448 